\title{
An Algorithm for Idle-State Detection in Motor-Imagery-Based Brain-Computer Interface
}

\author{
Dan Zhang, Yijun Wang, Xiaorong Gao, Bo Hong, and Shangkai Gao \\ Department of Biomedical Engineering, School of Medicine, Tsinghua University, Beijing 100084, China
}

Correspondence should be addressed to Yijun Wang, wyj97@mails.tsinghua.edu.cn

Received 28 February 2007; Accepted 27 May 2007

Recommended by Andrzej Cichocki

\begin{abstract}
For a robust brain-computer interface (BCI) system based on motor imagery (MI), it should be able to tell when the subject is not concentrating on MI tasks (the "idle state") so that real MI tasks could be extracted accurately. Moreover, because of the diversity of idle state, detecting idle state without training samples is as important as classifying MI tasks. In this paper, we propose an algorithm for solving this problem. A three-class classifier was constructed by combining two two-class classifiers, one specified for idle-state detection and the other for these two MI tasks. Common spatial subspace decomposition (CSSD) was used to extract the features of event-related desynchronization (ERD) in two motor imagery tasks. Then Fisher discriminant analysis (FDA) was employed in the design of two two-class classifiers for completion of detecting each task, respectively. The algorithm successfully provided a way to solve the problem of "idle-state detection without training samples." The algorithm was applied to the dataset IVc from BCI competition III. A final result with mean square error of 0.30 was obtained on the testing set. This is the winning algorithm in BCI competition III. In addition, the algorithm was also validated by applying to the EEG data of an MI experiment including "idle" task.
\end{abstract}

Copyright (c) 2007 Dan Zhang et al. This is an open access article distributed under the Creative Commons Attribution License, which permits unrestricted use, distribution, and reproduction in any medium, provided the original work is properly cited.

\section{INTRODUCTION}

People who suffer from severe motor disabilities but are still cognitively intact, need an alternative method to interact with the environment. Over the past decades, the development of the technology called brain-computer interface (BCI) has provided a novel and promising communication channel for these patients. A BCI is a communication system in which messages or commands that an individual wishes to convey to the external world do not pass through the brain's normal motor output pathways [1]. A BCI system can "read out" the intention of the patients and translates it into physical commands which control devices that serve the patients.

There are various BCI systems using different methods to extract the subjects' intentions from their EEG signals. One of the practical BCI systems is based on motor imagery (MI) $[2,3]$. The advantage of this type of BCI systems is that no external stimulation is needed. Current development of MI-based BCI is focused on how to discriminate different MI tasks and many algorithms could be ap- plied to get satisfied results. However, during practical use of BCI system, users may stay free of MI tasks (i.e., "idle state") at all. In order to make the system robust, the BCI system should be able to effectively detect the "idle state" and act properly. Moreover, because idle state may refer to various brain activities except the specific MI tasks, so it is not possible to acquire representative training samples for classifier designing. Therefore, to develop a new algorithm which cannot only discriminate different MI tasks but also effectively detect the idle state without any training samples is critical for improving present MI-based BCI system.

In this paper, an algorithm which integrates two twoclass classifiers with different parameters into one three-class classifier is proposed to overcome the difficulties mentioned above. The algorithm was applied to dataset IVc of BCI competition III. A final result with mean square error of 0.30 was obtained. In addition, an EEG experiment was carried out with similar setting as the one for the dataset of BCI competition III; the results showed the effectiveness of the proposed algorithm. 


\section{METHODOLOGY}

\subsection{Data description}

\subsubsection{Dataset IVc of BCl competition III}

BCI competitions are organized in order to foster the development of improved BCI technology by providing an unbiased validation of a variety of data-analysis techniques. The datasets of brain signals recorded during BCI experiments were from leading laboratories in BCI technology. Each data set is split into two parts: one part of labeled data ("training set") and another part of unlabeled data ("test set"). Researchers worldwide could tune their methods to the training data and submit the output of their translation algorithms for the test data.

Dataset IVc of BCI competition III was recorded from one healthy subject. The training dataset consisted of 3 sessions (70 trials for each session). Visual cues (letter presentation) indicated for 3.5 seconds which of the following 2 motor imageries the subject should perform: left hand, right foot. The presentations of target cues were intermitted by periods of random length, 1.75 to 2.25 seconds, in which the subject could relax. The testing data (6 sessions, 70 trials each) was recorded more than 3 hours after the training data. The experimental setup was similar to the training sessions, but the motor imagery had to be performed for 1 second only, compared to 3.5 seconds in the training sessions. The intermitting periods ranged from 1.75 to 2.25 seconds as before. The other difference was that a new task relax was added (also with visual cues as indications). The subject was required not to perform any MI tasks during relax task. 118-channel EEG signals were recorded during the experiment with sampling rate of $1000 \mathrm{~Hz}$ (see [4] for more details).

Competitors of this data set were required to classify a set of single-trial electroencephalograph (EEG) data recorded from three-class tasks in the testing set. The output of the classification must be a real number between -1 and 1 (ideally, -1 for left hand, 0 for relax, and 1 for right foot). The challenge was that the training set consists of only two-class data (left hand and right foot). One problem existed for the classification is that the testing set contains a new class relax with no training data. And there are two other problems: (1) the MI tasks in the testing set were performed for only $1 \mathrm{sec}$ ond instead of 3.5 seconds as in the training set; (2) the testing data was recorded more than 3 hours after the training data was acquired, so the distribution of some EEG features could be effected by long-term nonstationarities. All of these are practical problems in current MI-based BCI systems. The main difficulty is detecting an additional state relax without training samples, which is the same as "idle state" we mentioned in the previous section.

\subsubsection{Datasets from our Ml experiments}

The data set provided by BCI competition III was acquired from only one subject and the details of the experiment were not so clear. In order to thoroughly investigate the effective- ness of our algorithm, an MI experiment was carried out with a similar paradigm.

Three right-handed volunteers (two females and one male, 22 to 24 years old) participated in this experiment. There were three kinds of tasks in the experiment: left hand, right hand, and relax. Left hand and right hand referred to two MI tasks; while the subject was required not to perform any MI tasks during relax period. The subject was informed about which task to be performed by a visual cue on a PC screen before each trial. The trials lasted for 4 seconds with intermitting period of 2 seconds. 32-channel EEG (ActiveTwo system, BioSemi Instrumentation, Netherland) was recorded at the scalp over the motor cortex areas with a sampling rate of $256 \mathrm{~Hz}$. For every subject, 50 trials for each task were collected.

Compared to the data set of BCI competition III, "relax with no training data" was emphasized while the other issues were ignored: the tasks were performed for 4 seconds instead of 3.5 seconds/ 1 second and all trials were performed continuously. The purpose of increasing trial time was to improve the performance because it was difficult to get satisfied results for normal subjects in such a short time as 1 second. And long-term nonstationarities were not concerned here for the complexities and characteristics of MI tasks.

\subsection{Feature selection}

Motor imagery can be seen as mental rehearsal of a motor act without any obvious motor output [2]. It is broadly accepted that mental imagination of movements involves similar EEG patterns that are also found in real movements. The main difference between real movements and MI is that execution would be blocked at some corticospinal level in the latter case [3]. Recent studies show that when performing motor imagination, mu $(8-12 \mathrm{~Hz})$ and beta $(18-26 \mathrm{~Hz})$ rhythms are found to reveal event-related synchronization and desynchronization (ERS/ERD) over sensorimotor cortex just like when one actually does the motor tasks [5].

Event-related desynchronization (ERD) represents power decrease in given frequency bands of the ongoing EEG activity [5]. Preparation of movement is typically accompanied by ERD in mu and beta rhythms over somatosensory or motor cortex. Figure 1 displays the averaged ERD spatial mappings of the two MI tasks in the training set. We use the ratio of power decrease in the imagery state and the power in the rest state as the quantification of ERD [5]. The brain regions containing significant ERD over motor cortex are marked as A1 and A2 in Figure 1. The ERD of right-foot imagery exists in the central area (A2) while the ERD of left hand is localized in both hemispheres (A1) with contralateral dominance. This difference is the basis for classifying left-hand and right-foot imageries.

The mental state of relax differs substantially from those of left hand and right foot since no brain activity patterns similar with MI is evoked. It is reasonable to assume that during a relax task there is no obvious ERD over somatosensory or motor cortex. So relax status can be distinguished from left hand and right foot. Left hand can be recognized by existence 


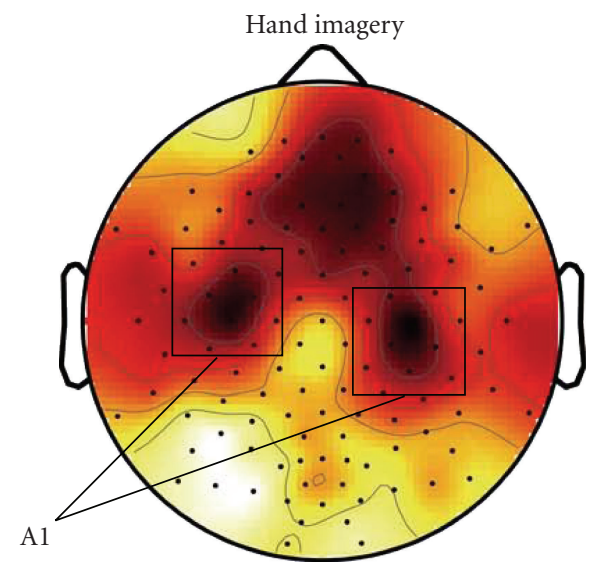

(a)
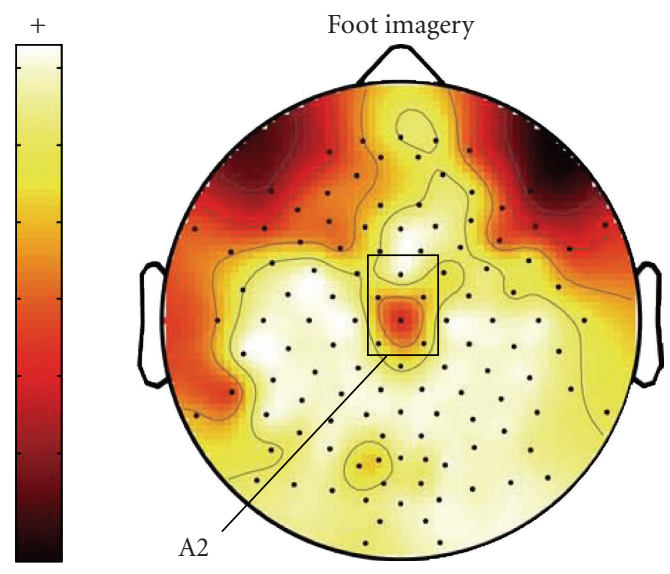

(b)

FIGURE 1: Averaged ERD spatial mappings of (a) left hand and (b) right foot in the training set.

of ERD in A1 area and right-foot is corresponding to the brain state with ERD in A2 area, while relax is just the brain state with no ERD in either A1 or A2 areas.

\subsection{Feature extraction}

The signals specific to the tasks are usually accompanied by interferences (such as noise, spontaneous EEG and other nontask activities). The common spatial subspace decomposition (CSSD) proposed by Wang et al. [6] was employed to extract the task-related source activities and to eliminate the background activities. The purpose of this method is to construct spatial filters which can distinguish two classes of signals based on simultaneous diagonalization of their covariance matrices [7].

In our method, we selected 37 EEG channels according to ERD distribution (see Figure 1), so only brain regions A1 and $\mathrm{A} 2$ are taken into consideration. Then we used the selected single-trial EEG data as the input matrix $X$ with 37 (channels) by 280 (samples, $0.71-3.50$ seconds) to construct spatial filters $\mathrm{SF}_{H}$ and $\mathrm{SF}_{F}$ for left hand and right foot, respectively. The spatial covariance of the EEG data can be obtained from

$$
C=X \cdot X^{T}
$$

The spatial covariance of each class is calculated as $C_{H}$ and $C_{F}$ by averaging over the trials in the corresponding class. The sum covariance matrix $C_{\text {Sum }}$ is factorized into the product of eigenvectors and eigenvalues

$$
C_{\text {Sum }}=C_{F}+C_{H}=U_{0} \cdot \Sigma \cdot U_{0}^{T} .
$$

The eigenvalues are assumed to be sorted in descending order. The whitening transformation matrix is then formed as

$$
P=\Sigma^{-1 / 2} \cdot U_{0}^{T}
$$

If $C_{H}$ and $C_{F}$ are transformed as

$$
S C_{F}=P \cdot C_{F} \cdot P^{T}, \quad S C_{H}=P \cdot C_{H} \cdot P^{T},
$$

then $C_{H}$ and $C_{F}$ share common eigenvectors and the sum of the corresponding eigenvalues for the two matrices will always be 1 , that is,

$$
S C_{F}=U \cdot \Sigma_{F} \cdot U^{T}, \quad S C_{H}=U \cdot \Sigma_{H} \cdot U^{T}, \quad \Sigma_{F}+\Sigma_{H}=I .
$$

As the sum of two corresponding eigenvalues is always one, the eigenvector with largest eigenvalue for $S_{F}$ has the smallest eigenvalue for $S_{H}$. This transformation is an effective way for separating variances in the two matrices $C_{H}$ and $C_{F}$. Taking out the first $m_{F}$ eigenvectors from $U$ as $U_{F}$ and the last $m_{H}$ eigenvectors from $U$ as $U_{H}$, the spatial filters for class $F$ and class $H$ are

$$
\mathrm{SF}_{F}=\left(U_{F}\right)^{T} \cdot P, \quad \mathrm{SF}_{H}=\left(U_{H}\right)^{T} \cdot P
$$

The eigenvectors left in $U$ correspond to the common spatial subspace of the two classes. The task-related components $S_{H}$ and $S_{F}$ are estimated by

$$
S_{F}=\mathrm{SF}_{F} \cdot X, \quad S_{H}=\mathrm{SF}_{H} \cdot X
$$

$X$ is a recorded data matrix of multichannel singletrial EEG. The columns of $\mathrm{SF}_{F}^{-1} / \mathrm{SF}_{H}^{-1}$ are spatial patterns corresponding to right-foot/left-hand components as timeinvariant EEG source distribution vectors [8].

The features used for classification are obtained by decomposing the EEG using $\mathrm{SF}_{F}$ and $\mathrm{SF}_{H}$. The feature vectors of one single trial are defined as

$$
\begin{aligned}
& f_{H, i}=\log \left(\operatorname{var}\left(S_{H, i}\right)\right), \quad i=1, \ldots, m_{H}, \\
& f_{F, i}=\log \left(\operatorname{var}\left(S_{F, i}\right)\right), \quad i=1, \ldots, m_{F} .
\end{aligned}
$$


$S_{H, i} / S_{F, i}$ represents the $i$ th row vector of $S_{H} / S_{F}$. The $\log$ transformation serves to approximate normal distribution of the data. Our experiences on the training set indicated that setting $m_{F}=3$ and $m_{H}=3$ was enough to get a fairly good performance.

During left-hand imagery, ERD occurs in region A1, leading to a relatively decreased EEG variance in this area. Therefore, right foot has a higher EEG variance than left hand in region A1. This behavior is reflected by large coefficients for channels covering region A1 in the spatial pattern corresponding to right-foot imagery. Figure 2 displays the most important spatial pattern of the two tasks. As shown in Figure 2(b), the most important spatial pattern of right foot accords with the ERD distribution of left hand. The spatial filter $\mathrm{SF}_{F}$ serves as extracting the component with a source distribution like the corresponding spatial pattern. Therefore, the component extracted by $\mathrm{SF}_{F}$ can be considered as the source activity concerning left-hand $\mathrm{ERD}$, which has a significant distribution over region A1. A weak source activity leads to a small variance of relative scalp EEG, which is corresponding to significant ERD. Due to no ERD in region A1, the component of right foot has a larger variance than that of left hand when filtered by $\mathrm{SF}_{F}$, that is,

$$
\operatorname{var}\left(\mathrm{SF}_{F} \cdot X_{F}\right)>\operatorname{var}\left(\mathrm{SF}_{F} \cdot X_{H}\right) \text {, }
$$

where $X_{H}$ and $X_{F}$ are single-trial EEG corresponding to left hand and right foot, respectively. We can also get another inequality as follows:

$$
\operatorname{var}\left(\mathrm{SF}_{H} \cdot X_{H}\right)>\operatorname{var}\left(\mathrm{SF}_{H} \cdot X_{F}\right) .
$$

Note that according to the above definitions, left-hand MI causes a relatively increased EEG variance over area A2 (corresponding to right-foot task) because event-related desynchronization of EEG takes place on area A1. This behavior is reflected in large coefficients for electrodes on area A2 in the spatial filter of left-hand $\left(\mathrm{SF}_{H}\right)$ [8], and vice versa for right foot.

\subsection{Classification method}

The paper of Garrett et al. [9] showed that if features were properly extracted, the performance of linear classifiers can behave as well as that of complex nonlinear classifiers, so we simply used Fisher discriminant analysis (FDA) in our method.

After using CSSD to extract ERD feature out of the training set, FDA was applied for classification and an accuracy of $(99.1 \pm 1.2) \%$ was obtained on the training set using a $10 \times 10$ fold cross-validation. The result of FDA proves that there is no need to use other complicated methods.

\subsection{Classification on the testing set}

Denote $X_{R}$ as a single-trial EEG of relax, as no ERD occurs in both regions $\mathrm{A} 1$ and $\mathrm{A} 2$ during relax tasks, the following inequalities come into existence:

$$
\begin{aligned}
& \operatorname{var}\left(\mathrm{SF}_{F} \cdot X_{R}\right)>\operatorname{var}\left(\mathrm{SF}_{F} \cdot X_{H}\right), \\
& \operatorname{var}\left(\mathrm{SF}_{H} \cdot X_{R}\right)>\operatorname{var}\left(\mathrm{SF}_{H} \cdot X_{F}\right) .
\end{aligned}
$$

Both components of relax and right foot are larger than that of left hand when filtered by $\mathrm{SF}_{F}$, so left-hand motor imageries can be discriminated from right-foot/relax. Similarly, right foot can be discriminated from left-hand/relax when filtered by $\mathrm{SF}_{H}$.

The required classification outputs of left hand and right foot are defined as -1 and +1 . If we do a two-class classification based on the feature vectors $f_{H}$ extracted by $\mathrm{SF}_{H}$ and set the classification outputs of left hand and right foot to -1 and +1 as required, then samples of relax are also classified to -1 as it is the same as left hand according to (9) and (11). Samples of relax are classified to +1 according to (10) and (12). Table 1 shows the different outputs of the three tasks in ideal conditions. Column " $f_{F}$ " and " $f_{H}$ " shows the two two-class classification results. Column " $\left(f_{F}+f_{H}\right) / 2$ " represents the mean value of two outputs corresponding to $f_{F}$ and $f_{H}$ in the same row. Ideally, the two classifiers corresponding to " $f_{F}$ " and " $f_{H}$ " will result in opposite outputs for relax $(+1 /-1)$ and the final classification result of relax can be set to 0 easily by " $\left(f_{F}+f_{H}\right) / 2$." Therefore, it is possible to separate the three classes.

Our strategy goes as following: at first, a two-class classifier was used to classify samples of relax to output 0 (see Table 1). Then the second two-class classifier was defined to classify the remaining samples into either right foot or left hand. The whole procedure of the classification algorithm is shown in Figure 3.

Step 1 (Discriminating the relax trials). The classification process of this step is showed in Figure 4. A subject-specific bandpass filter of $12-14 \mathrm{~Hz}$ (with most significant ERD feature for the subject of dataset IVc) and a time window of $0.71-3.50$ seconds (eliminating the first 0.7 seconds as reaction time) were set for CSSD algorithm to calculate two spatial filters $\mathrm{SF}_{F 1}$ and $\mathrm{SF}_{H 1}$ from the training data. Because the duration of each trial in the testing set is much shorter than that of the training set, CSSD filter cannot get enough information with such a short time window to keep a high accuracy. Here we bring forward another assumption that the spatial pattern in the intermitted time (1.75-2.25 seconds) after a relax trial is similar to that of the relax trial (however, the intermitted time after an MI task cannot be simply considered as relax because the subject might keep on doing MI for a certain period even after he saw the cue for stop). With this assumption, a time window of 2.75 seconds ( 1 second for the task and at least 1.75 seconds for intermitted time) was selected as the input of the CSSD filters for the testing set. The effective duration of relax can thus be prolonged, making the classification results more reliable than those obtained by only using a short-time window.

Bagging strategy [10] was used here for reducing variance of classification accuracy. 160 trials were randomly selected 


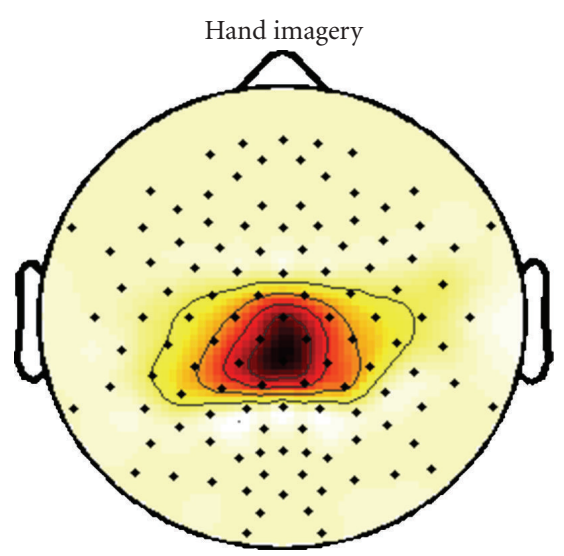

(a)
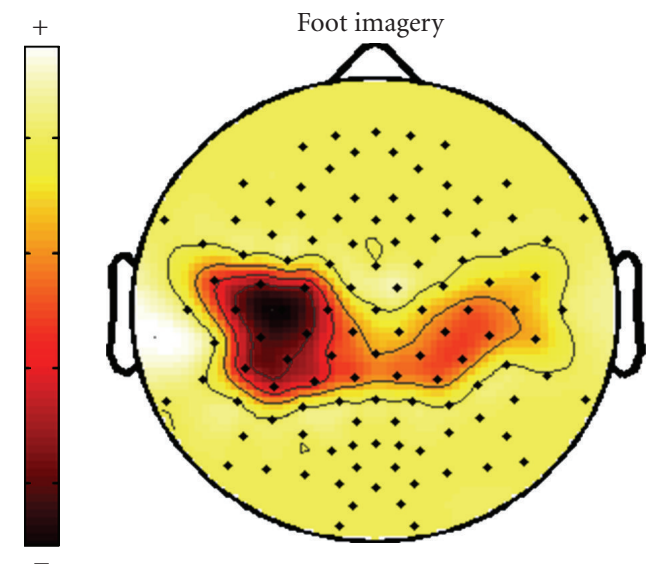

(b)

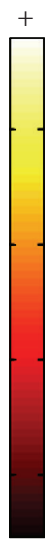

FIGURE 2: Most important spatial pattern of (a) left hand and (b) right foot.

TABLE 1: Ideal classification results of the three tasks.

\begin{tabular}{l|ccc}
\hline Feature task & $f_{F}$ & $f_{H}$ & $\left(f_{F}+f_{H}\right) / 2$ \\
\hline Left hand & $-1($ ERD in A1) & $-1($ no ERD in A2) & $-1(-1 /-1)$ \\
Right foot & $+1($ no ERD in A1) & $+1($ ERD in A2) & $+1(+1 /+1)$ \\
Relax & $+1($ no ERD in A1) & $-1($ no ERD in A2) & $0(+1 /-1)$ \\
\hline
\end{tabular}

" $\left(f_{F}+f_{H}\right) / 2$ " represents the mean value of two outputs corresponding to $f_{F}$ and $f_{H}$ in the same row.

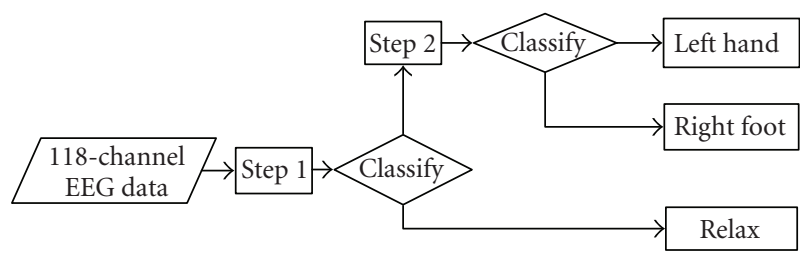

Figure 3: Flow chart of our algorithm.

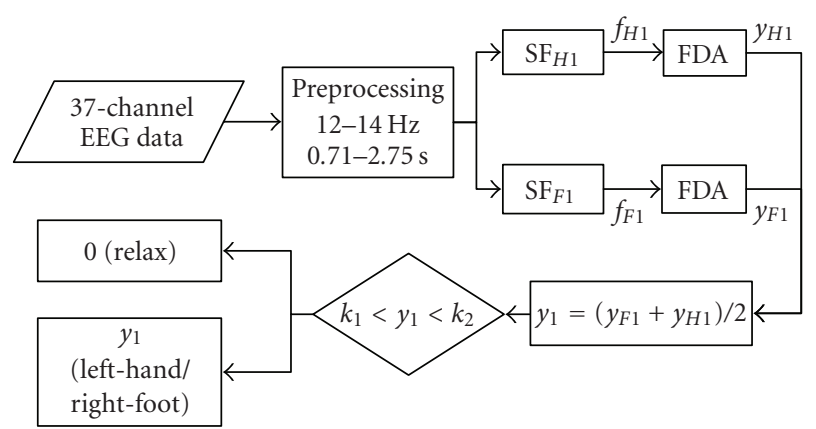

Figure 4: Classification process of Step 1.

out of all 210 trials in the training set to derive a classifier which was applied on each trial in the testing set. This process was repeated for 100 times, of which the classification outputs were averaged to get the final result. As shown in
Figure 4 there are two FDA classifiers following two spatial filters $\mathrm{SF}_{F 1}$ and $\mathrm{SF}_{H 1}$. The outputs of these two classifiers $\left(y_{F 1}\right.$ and $\left.y_{H 1}\right)$ were normalized to real number between -1 and 1 and were averaged to get a higher classification accuracy [11]. In Step 1 the averaging also has an effect of setting relax to 0 .

After classification, two thresholds (upper boundary above 0 and lower boundary below 0 ) were determined manually, according to the distribution of training samples. The samples with classification outputs near 0 were labeled as relax. The remaining samples are left unlabeled to be fed as the input of Step 2. The process is shown as following where $k_{1}$ and $k_{2}$ denote the two thresholds:

$$
z= \begin{cases}0, & \text { if } k_{1}<y_{1}<k_{2}, \\ y_{1}, & \text { if } y_{1}<k_{1} \text { or } y_{1}>k_{2}\end{cases}
$$

In our algorithm, we propose these two thresholds could be chosen to make $P_{1}$ (in percentage) of the trials of MI tasks with nonzero classification output. ( $P_{1}$ was set to $70 \%$ for the results submitted to dataset IVc based on our former experiences)

Step 2 (Discriminating the remaining trials). Step 1 is good for picking out relax but not optimal for classifying left hand and right foot because the intermitted time has been taken into consideration. During the intermitted time after left hand and right foot, the subject is told to "relax." So a short time window (0.61-1.20 seconds) was defined as for this step. Besides, our investigation showed that a widepass band for 
temporal filtering $(11-27 \mathrm{~Hz})$ was better for classifying left hand and right foot. This wider frequency band including both mu and beta band is also good for generalization. The same time window as in Step 1 (0.71-3.50 seconds) was applied to calculate $\mathrm{SF}_{\mathrm{F} 2}$ and $\mathrm{SF}_{\mathrm{H} 2}$ with the training set.

The classification process of this step is shown in Figure 5. After classification we also set two thresholds manually to label samples with outputs congregating near -1 and 1 to right hand and left foot, respectively, and the others to a real number between -1 and 1 . The normalization process is as follows, where $y$ is the original output and $z$ is the normalized output, $k_{3}$ and $k_{4}$ denote the two thresholds:

$$
z= \begin{cases}-1, & \text { if } y_{2}<k_{3}, \\ -\frac{y_{2}}{k_{3}}, & \text { if } k_{3} \leq y_{2} \leq 0 \\ \frac{y_{2}}{k_{4}}, & \text { if } 0 \leq y_{2} \leq k_{4} \\ 1, & \text { if } y_{2}>k_{4} .\end{cases}
$$

In our algorithm, we propose these two thresholds could be selected to make $P_{2}$ (in percentage) of trials of MI tasks with classification outputs of \pm 1 . ( $P_{2}$ was set to $70 \%$ for the results submitted to Data Set IVc).

For the data from our MI experiments, a time window of 0.5-4 seconds was applied to calculate spatial filters for both Steps 1 and 2. The frequency band used in Step 1 was subjectspecific and $11-27 \mathrm{~Hz}$ were chosen in Step 2. Half samples of MI tasks (25 trials for left hand, 25 trials for right hand) were employed in the training set while the rest were used as the testing set. By randomly selecting trials for training, the classification process was repeated for 50 times to get average results. Features were extracted from both task and intermitting periods (6 seconds) in Step 1 while only task periods were considered in Step 2. Furthermore, we investigated how to choose threshold $k_{1}-k_{4}$ to get a better performance (refer to Section 3 for details).

\section{RESULTS}

The result of dataset IVc was evaluated by mean square error criterion. Defining the true labels for 420 trials in the testing set as $y_{1}, y_{2} \cdots y_{420}$, and the classification outputs as $z_{1}, z_{2} \cdots z_{420}$, the mean square error (MSE) was calculated as

$$
\text { MSE }=\frac{1}{420} \sum_{i=1}^{420}\left(y_{i}-z_{i}\right)^{2} .
$$

As the winning algorithm in BCI competition III, a mean square error of 0.30 was achieved by our algorithm, which was much lower than the result of the second best competitor, who achieved 0.59 [12]. Figure 6 shows the distribution of the classification results of the three classes. Approximately $60 \%$ samples of true left hand and right foot are correctly classified to -1 and 1 , and about $40 \%$ of relax samples are classified to 0 . The particular strength of this method was that it managed to identify nearly half of the relax trials and none of the other submissions to this dataset han- dled the idle state well even if they discriminate the two MI tasks as well as our algorithm [12]. This could be the evidence that traditional algorithms are not so effective for classifying idle state. The results proved the effectiveness of this algorithm.

MSE is a specific performance measure used in BCI competition III. Two other measures with more direct meaning are defined as below.

\section{(a) Probability of detection (POD)}

For a certain task A, considering all trials of task A, let $N_{D}$ denote the number of trials correctly detected as task A, $N_{M}$ the number of trials missed, then POD is defined as

$$
\mathrm{POD}=\frac{N_{D}}{N_{D}+N_{M}} .
$$

POD represents the true positive rate of certain brain states. Two values were calculated based on POD: POD of MI tasks and POD of idle states (relax task). For POD of MI tasks, we only care if MI tasks could be discriminated from idle states. Whether MI tasks were classified correctly is another issue. For a practical BCI system, the POD of idle states is critical because false alarms during idle states may lead to unexpected action of the BCI system when the subjects are resting or idling.

\section{(b) Classification accuracy (CA)}

For a certain MI task A, considering all trials of task A, let $N_{C}$ denote the number of trials correctly classified as task A, $N_{W}$ the number of trials classified as other MI tasks, then CA is defined as

$$
\mathrm{CA}=\frac{N_{C}}{N_{C}+N_{W}} .
$$

According to this definition, the number of trials classified as idle states is not included in (17). It is easy to understand: failure of detection will not lead to execution of improper commands; only the average time for carrying out one command will be lengthened. From this point of view, the POD of MI tasks together with CA decides the speed of the synchronized BCI system. The mean CA value of both MI tasks was calculated as the average CA.

Referring to our proposed criterion for selecting thresholds, $k_{1}-k_{4}$ were decided by $P_{1}$ and $P_{2}$. Varying these two probabilities leads to changes of the performance measures defined above. Ideally, a BCI system with good performance is associated with CA, POD of MI tasks and idle states close to $100 \%$. Therefore, $P_{1}$ and $P_{2}$ should be chosen carefully to make the real BCI system with a good performance.

To simplify this problem, we make both $P_{1}$ and $P_{2}$ equal to a certain value $P$. Table 2 listed the results calculated by increasing $P$ from 0.6 to 1 in step of .05 for subject FL. The values of $\mathrm{CA}$ and $\mathrm{POD}_{\text {Idle }}$ are negative correlated with $P$, while $\mathrm{POD}_{\mathrm{MI}}$ is positive correlated with $P$. The basic principle for choosing $P$ value is to reduce false alarm during idle states (i.e., increase $\mathrm{POD}_{\mathrm{Idle}}$ ) while keeping $\mathrm{POD}_{\mathrm{MI}}$ and $\mathrm{CA}$ at an 


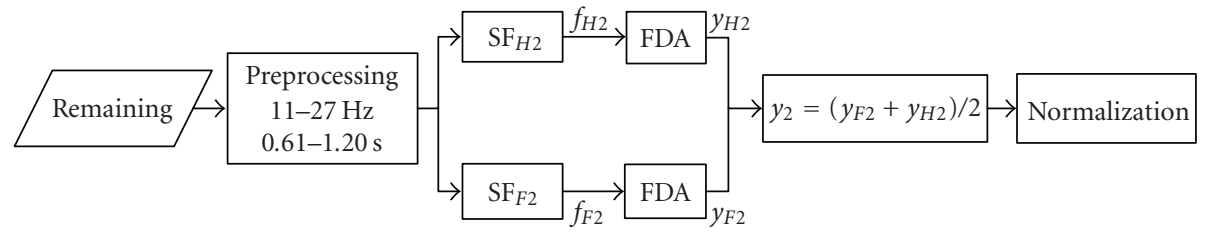

FIgURE 5: Classification process of Step 2.

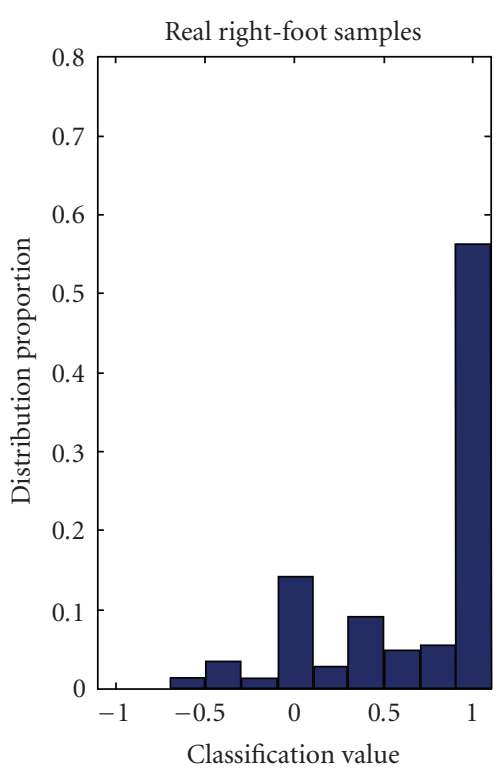

(a)

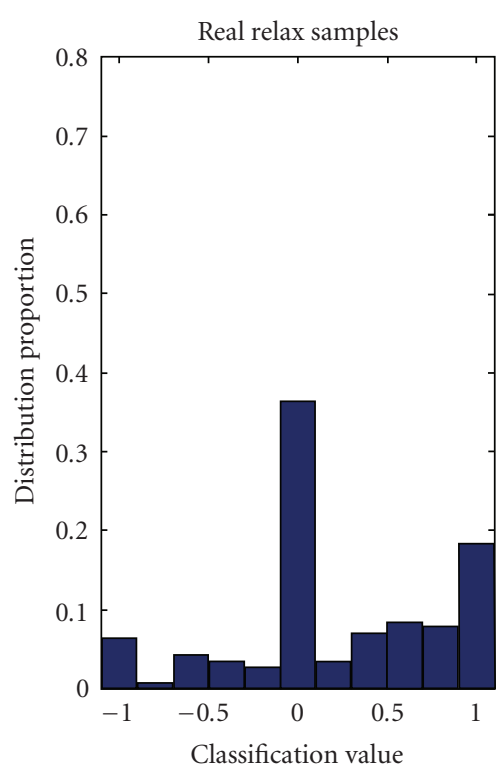

(b)

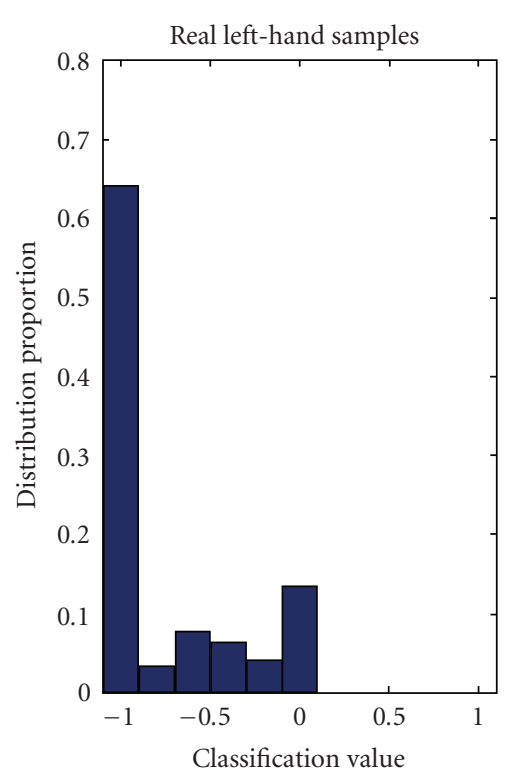

(c)

FIGURE 6: Distribution of classification results with respect to the three true labels.

acceptable level. The optimal $P$ value for subject FL is manually selected as 70\% with high $\mathrm{CA}$ and $\mathrm{POD}_{\text {Idle }}$ as well as a relatively high $\mathrm{POD}_{\mathrm{MI}}$ (see Table 2 ). In the same way, the optimal $P$ values for the other two subjects are chosen as $80 \%$ (ZD) and 90\% (ZYJ); the corresponding results are shown in Table 3. The data of all three subjects achieved nearly $100 \%$ CA for discriminating the two MI tasks, with average $\mathrm{POD}_{\mathrm{MI}}$ about $70 \%$ and average POD $_{\text {Idle }}$ above $90 \%$.

\section{CONCLUSIONS AND DISCUSSION}

The most important characteristic of our algorithm was combining two two-class classifiers to construct a three-class classifier. We broke down the problem into two steps and solved them consecutively with parameters separately optimized in each step for its own purpose. The analysis of the final result validated this strategy.

The basic assumption was that during relax task there is no obvious ERD over somatosensory or motor cortex. This assumption is shown to be reasonable according to the final results. Figure 7 displays the averaged spatial mapping of relax (calculated in a same way as in Figure 1) in the testing set. There is no obvious ERD in region A1 and A2. Figure 8 shows the classification results of the samples in the testing set by these two classifiers and the true labels are given by different legends. Most samples of relax are located in the second

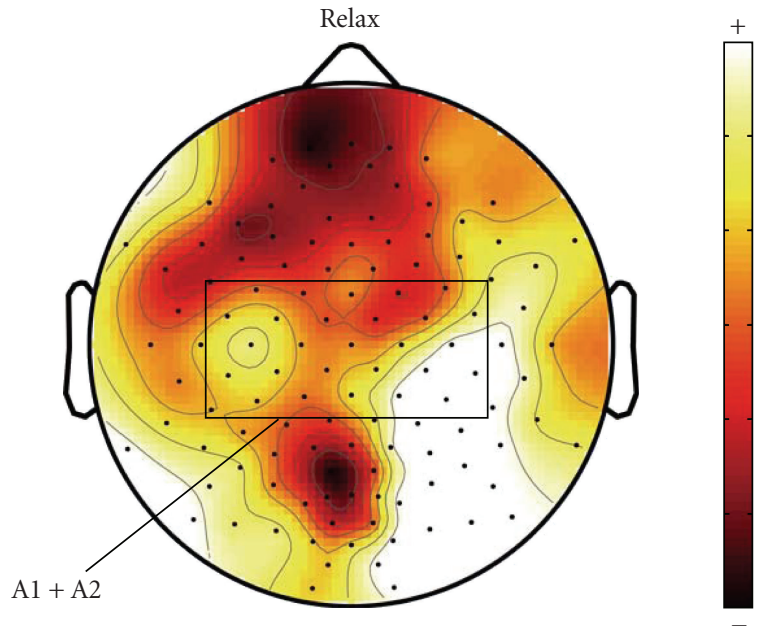

FIGURE 7: Averaged spatial mapping of relax (calculated in a same way as in Figure 1) in the testing set.

quadrant, while right-foot and left-hand samples are in the first and third quadrants. This distribution is in accordance with the analysis in Table 1.

In Section 2.1 we listed three problems in dataset IVc, our algorithm addressed the problem of no training data for re- 
TABle 2: Performance measures of subject FL corresponding to different $P$ values.

\begin{tabular}{lccc}
\hline$P\left(P_{1} \& P_{2}\right)$ & POD $_{\mathrm{MI}}$ & POD $_{\text {Idle }}$ & CA \\
\hline $100 \%$ & $100.0 \pm 0.0 \%$ & $0.0 \pm 0.0 \%$ & $89.0 \pm 2.3 \%$ \\
$95 \%$ & $96.1 \pm 1.8 \%$ & $4.2 \pm 1.2 \%$ & $94.9 \pm 1.8 \%$ \\
$90 \%$ & $90.0 \pm 1.6 \%$ & $61.2 \pm 2.1 \%$ & $96.8 \pm 1.1 \%$ \\
$85 \%$ & $84.2 \pm 2.3 \%$ & $71.0 \pm 3.2 \%$ & $97.2 \pm 2.5 \%$ \\
$80 \%$ & $74.1 \pm 1.9 \%$ & $81.4 \pm 1.8 \%$ & $96.6 \pm 2.1 \%$ \\
$75 \%$ & $65.3 \pm 2.2 \%$ & $91.0 \pm 1.6 \%$ & $97.6 \pm 1.4 \%$ \\
$70 \%$ & $62.7 \pm 3.2 \%$ & $95.5 \pm 0.9 \%$ & $98.9 \pm 0.8 \%$ \\
$65 \%$ & $51.8 \pm 2.0 \%$ & $98.1 \pm 2.2 \%$ & $98.7 \pm 1.0 \%$ \\
$60 \%$ & $45.1 \pm 1.6 \%$ & $99.6 \pm 0.9 \%$ & $99.3 \pm 1.2 \%$ \\
\hline
\end{tabular}

Table 3: Performance measures of three subjects with the optimal $P$ values.

\begin{tabular}{c|cccc}
\hline Subject & Optimal $P$ & POD $_{\mathrm{MI}}$ & POD $_{\text {Idle }}$ & CA \\
\hline ZYJ & $90 \%$ & $78.2 \pm 1.7 \%$ & $90.2 \pm 1.3 \%$ & $98.3 \pm 1.2 \%$ \\
FL & $70 \%$ & $62.7 \pm 3.2 \%$ & $95.5 \pm 0.9 \%$ & $98.9 \pm 0.8 \%$ \\
ZD & $80 \%$ & $61.2 \pm 2.2 \%$ & $96.1 \pm 1.1 \%$ & $99.4 \pm 0.4 \%$ \\
\hline
\end{tabular}

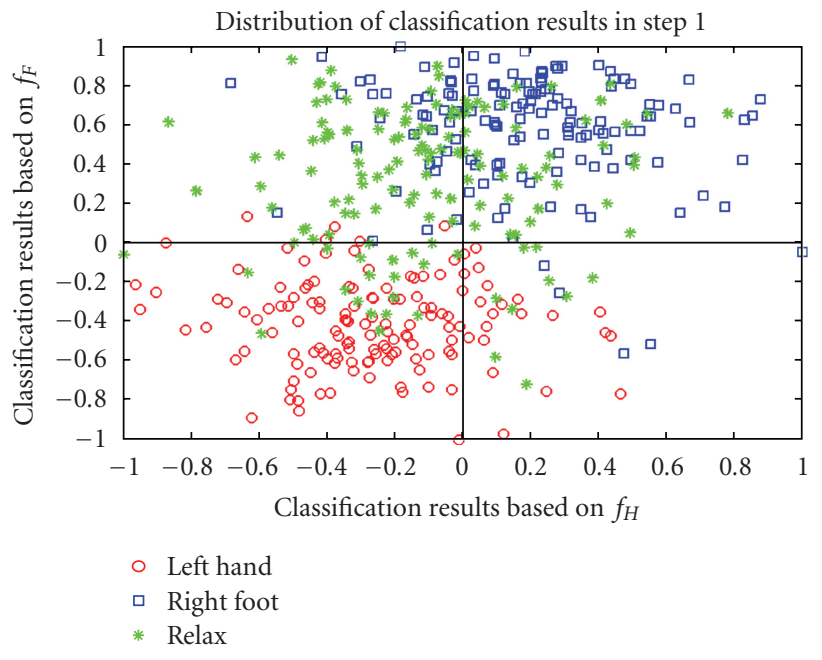

Figure 8: Distribution of classification results in Step 1.

lax. The other two problems may lead to nonsignificant interference with the application of CSSD algorithm, which is essentially determined by the spatial patterns of different MI tasks.

The problems of shortened trial time and long-term nonstationarities seem to be not so critical here because the two MI tasks still can be discriminated well (see Figure 6). One possible reason is because this data set is from a very good subject (classification accuracy on training set is around $99 \%$ ). For subjects with ordinary performance, the results might be worse. However, most subjects could achieve better performances after a certain period of training.

Another issue worth mentioning is the difference between relax and idle states. Relax might be slightly different from idle states, which are always referred to a quite long period with no MI going on rather than 1-second trials in these testing sessions. The brain states during relax trials in the testing sessions could be better described as "noncontrol" or "non-MI" states. In our algorithm, relax trials are only considered as brain states quite different from MI trials and no information were retrieved from these trials for designing the algorithm. From this point of view, we consider them as equal terms in this paper.

The traditional ways of idle-state detection mainly focus on developing powerful and robust algorithm mathematically. Our strategy aims at building a practical BCI system. In our opinion, how to integrate these methods in an effective way is also very important. Because the nature of idle states is quite different from those MI states, it is worth to set up an additional step with optimal parameters for separating these relax trials from the rest trials.

The proposed algorithm achieved satisfied results on our MI datasets. It shows the effectiveness of our algorithm for practical BCI systems. This result is also much better than dataset IVc of BCI competition III. The main reason might be due to the lengthened trial time, which is important for the subjects' performance.

The probabilities $\left(P_{1}\right.$ and $\left.P_{2}\right)$, which decide the thresholds $k_{1}-k_{4}$, are crucial to the performance of our algorithm. For dataset IVc, we simply select $70 \%$ for both $P_{1}$ and $P_{2}$ based on our former experiences. These $P$ values could be carefully chosen to make the performance better based on the three indexes $\left(\mathrm{POD}_{\mathrm{MI}}, \mathrm{POD}_{\text {Idle }}, \mathrm{CA}\right)$ defined above. Decreasing $P$ value will lead to higher POD $_{\text {Idle }}$ but lower POD $_{M I}$, which is the key factor for the speed of the synchronized BCI system. Also higher CA will be achieved because more ambiguous MI trials are labeled relax. Our current strategy is to insure a high POD $_{\text {Idle }}$ (i.e., above 90\%) first, and then make $\mathrm{POD}_{\mathrm{MI}}$ and $\mathrm{CA}$ as high as possible. We have not established an automatic way to make a balance between $\mathrm{POD}_{\mathrm{MI}}$ and $\mathrm{POD}_{\text {Idle }}$ yet. These results might be further improved by selecting optimal thresholds $k_{1}-k_{4}$ based on advanced statistical theories. 
A BCI system that can distinguish patterns not included in training data is very attractive. Solving the problem of dataset IVc is a good step towards this target. The proposed algorithm is especially useful to reduce the false alarms in current BCI system based on MI when the subjects are not performing MI tasks. Although we perform offline analysis here, this algorithm could be easily moved to online system.

\section{ACKNOWLEDGMENTS}

This project is supported by National Natural Science Foundation of China (no. 60318001, 30630022), and TsinghuaYuyuan Medicine Research fund. We are grateful to KlausRobert Müller, Benjamin Blankertz, and Gabriel Curio for providing the dataset. Also, we would like to extend our special thanks to Prof. Fusheng Yang for a very informative discussion and his valuable comments.

\section{REFERENCES}

[1] J. R. Wolpaw, N. Birbaumer, D. J. McFarland, G. Pfurtscheller, and T. M. Vaughan, "Brain-computer interfaces for communication and control," Clinical Neurophysiology, vol. 113, no. 6, pp. 767-791, 2002.

[2] G. Pfurtscheller and C. Neuper, "Motor imagery and direct brain-computer communication," Proceedings of the IEEE, vol. 89, no. 7, pp. 1123-1134, 2001.

[3] M. Jeannerod, "Mental imagery in the motor context," Neuropsychologia, vol. 33, no. 11, pp. 1419-1432, 1995.

[4] http://ida.first.fraunhofer.de/projects/bci/competition_iii/ desc_IVc.html.

[5] G. Pfurtscheller and F. H. Lopes da Silva, "Event-related EEG/MEG synchronization and desynchronization: basic principles," Clinical Neurophysiology, vol. 110, no. 11, pp. 1842-1857, 1999.

[6] Y. Wang, P. Berg, and M. Scherg, "Common spatial subspace decomposition applied to analysis of brain responses under multiple task conditions: a simulation study," Clinical Neurophysiology, vol. 110, no. 4, pp. 604-614, 1999.

[7] Y. Wang, Z. Zhang, Y. Li, X. Gao, S. Gao, and F. Yang, "BCI competition 2003-data set IV: an algorithm based on CSSD and FDA for classifying single-trial EEG," IEEE Transactions on Biomedical Engineering, vol. 51, no. 6, pp. 1081-1086, 2004.

[8] H. Ramoser, J. Müller-Gerking, and G. Pfurtscheller, "Optimal spatial filtering of single trial EEG during imagined hand movement," IEEE Transactions on Rehabilitation Engineering, vol. 8, no. 4, pp. 441-446, 2000.

[9] D. Garrett, D. A. Peterson, C. W. Anderson, and M. H. Thaut, "Comparison of linear, nonlinear, and feature selection methods for EEG signal classification," IEEE Transactions on Neural Systems and Rehabilitation Engineering, vol. 11, no. 2, pp. 141144, 2003.

[10] L. Breiman, "Bagging predictors," Machine Learning, vol. 24, no. 2, pp. 123-140, 1996.

[11] G. Dornhege, B. Blankertz, G. Curio, and K.-R. Müller, "Boosting bit rates in noninvasive EEG single-trial classifications by feature combination and multiclass paradigms," IEEE Transactions on Biomedical Engineering, vol. 51, no. 6, pp. 993 1002, 2004.
[12] B. Blankertz, K.-R. Müller, D. J. Krusienski, et al., “The BCI competition III: validating alternative approaches to actual BCI problems," IEEE Transactions on Neural Systems and Rehabilitation Engineering, vol. 14, no. 2, pp. 153-159, 2006. 

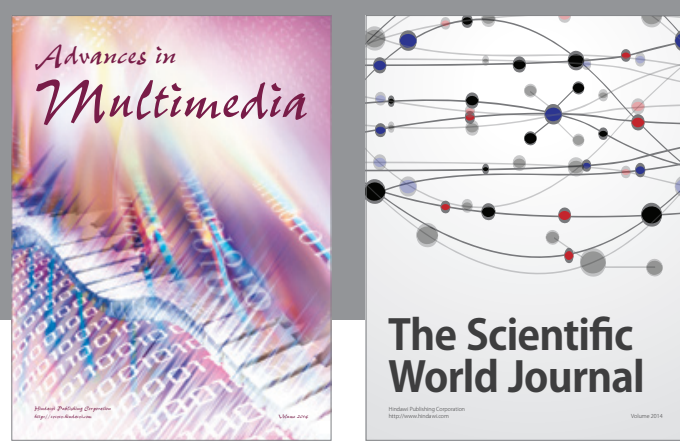

The Scientific World Journal
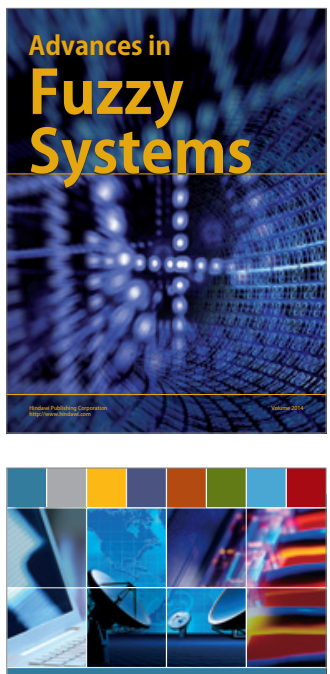

Computer Networks and Communications
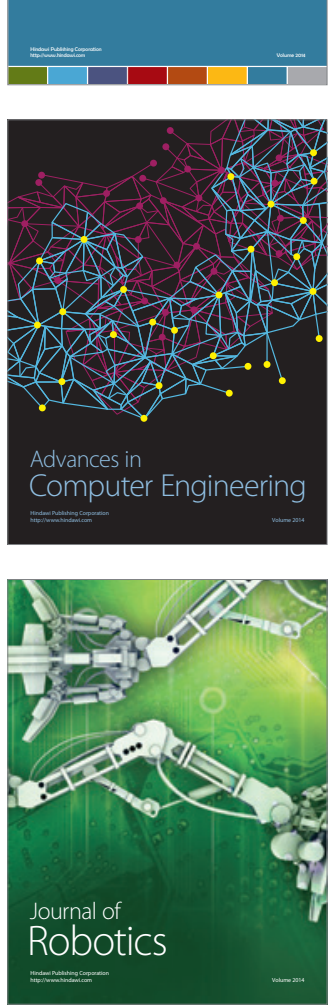
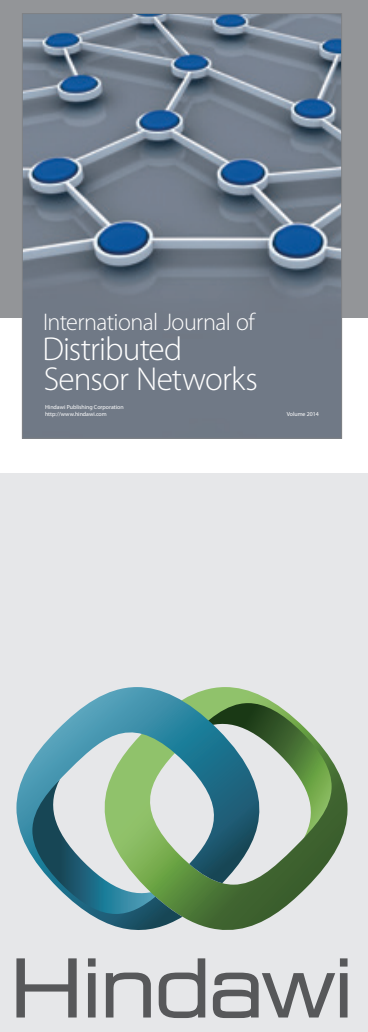

Submit your manuscripts at

http://www.hindawi.com
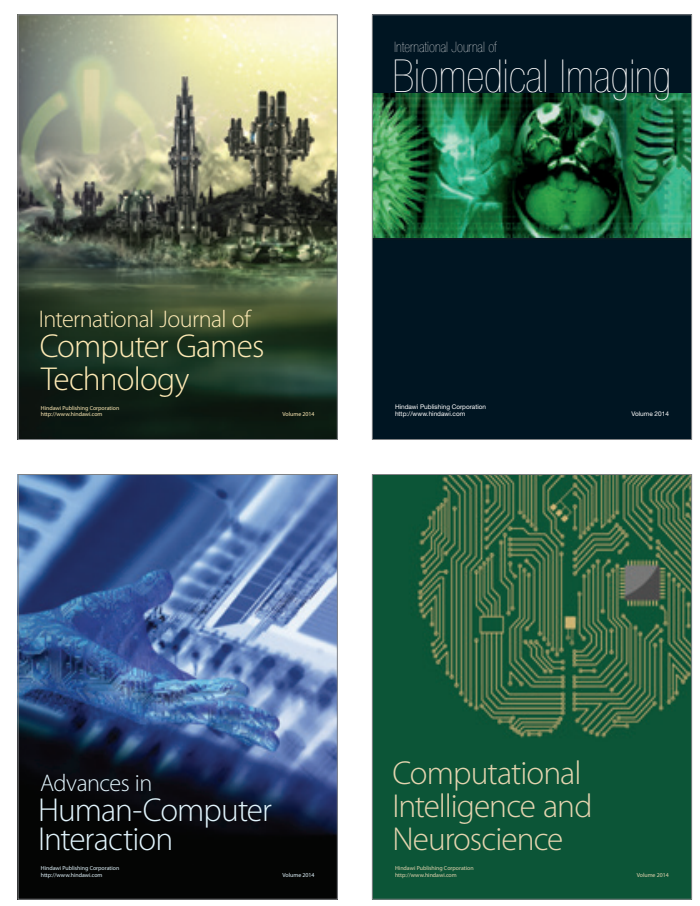
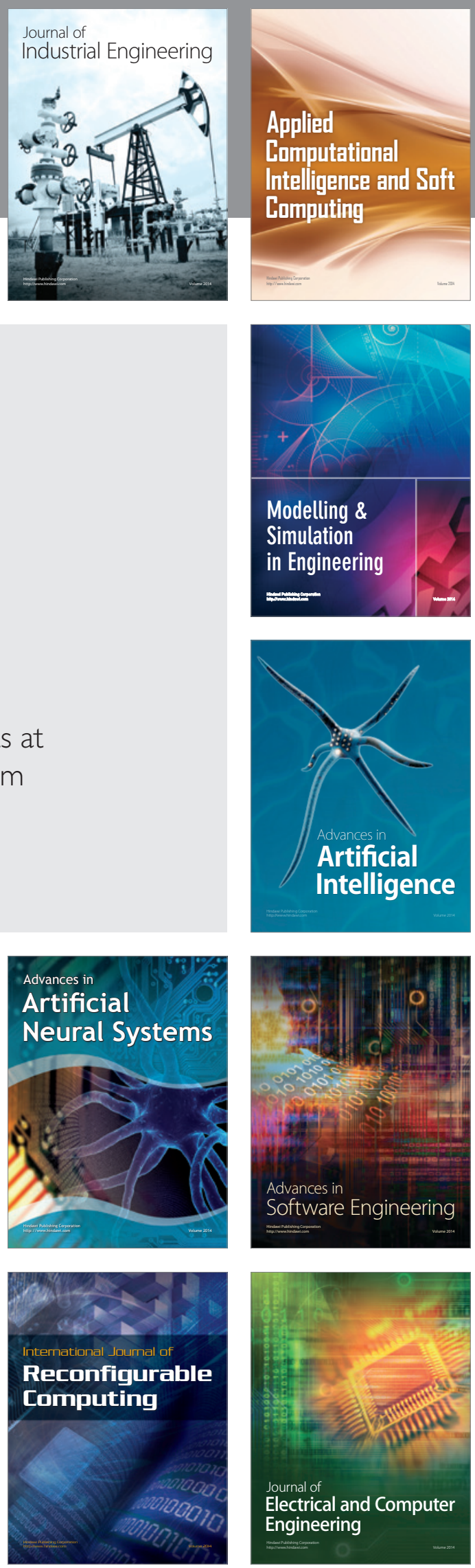